\title{
The Impact from Comorbid Diseases on Compliance in Children
}

\author{
Tatiana V. Stoeva, MD, Elena V. Titkova, PhD, Olga N. Nikolaychuk, PhD, Olga V. Jagiashvili, \\ Olesya V. Reshetilo, Victoriya V. Syitnik
}

\begin{abstract}
Comorbid pathology in children significantly reduces the quality of life, prevents full of compliance to therapy, provokes worsening of the general condition of the child. Combination of disease, expensive treatment may result decreased adherence therapy quality of care that ultimately may affect on the prognosis of the disease. The aim of our study was to detect communication between comorbid diseases in children and the level of compliance to therapy. Atopic dermatitis(AD) is often accompanied by comorbid diseases. The presence of comorbid diseases in children accompanied by more severe AD. Compliance in a group of comorbid disease was significantly lower. Among the reasons for low adherence to treatment can be identified: the duration and lack of effectiveness of the treatment, the complexity of the use of drugs, number of drugs and various forms of drugs that had to apply for the relief of the underlying disease, and treatment of comorbidity.
\end{abstract}

Key words: atopic dermatitis, adherence therapy quality, comorbid pathology.

\section{INTRODUCTION}

In modern conditions, many diseases in children are characterized by a combination of defeat. The disease process often involved several organs and even systems. The frequency of this combination is greater than the probability of a random match. Comorbid pathology in children significantly reduces the quality of life, prevents full of compliance to therapy and provokes worsening of the general condition of the child. Thus, for example, atopic dermatitis (AD) in children in itself, beginning at an early age, takes a chronic course, significantly worsens the quality of life, causing serious family stress [2]. Sleep disturbance, loss of a job, the time needed to care for patients with $\mathrm{AD}$ and considerable financial costs, including direct costs of treatment and indirect costs of job loss - all these factors contribute to the emergence of family stress. In this situation, it should be emphasized mental suffering of the patient. AD leads to a change in the

Tatiana V. Stoeva MD, professor, Odessa National Medical University Ukraine

Elena V. Titkova, PhD, Assistant Professor, Odessa National Medical University Ukraine elena-titkova@ yandex.ru

Olga N. Nikolaychuk, PhD, Associate Professor, Odessa National Medical University, Ukraine

Olga V. Jagiashvili, Assistant Professor, Odessa National Medical University , Ukraine

Olesya V. Reshetilo, Assistant Professor, Odessa National Medical University, Ukraine

Victoriya V. Syitnik, Assistant Professor, Odessa National Medical University, Ukraine appearance, reduce confidence in the collective adaptation, disruption of psychosexual development of the personality, irritability, fearfulness, anxiety, depression and introvert. The need for significant restrictions in the diet and daily life, daily round specialized skin care, a significant reduction in quality of life during exacerbation of the disease, the possibility of existence of such pathologies as allergic rhinitis and asthma, dissatisfaction with the results of treatment - all this places a heavy burden on the patient and his family. Often, these children have comorbid pathology. So, AD is the first clinical manifestation of "atopic march" and a significant risk factor for the formation of allergic rhinitis and asthma in children. In addition, these children have more severe asthma than patients who initially suffered from asthma only [1]. This combination of disease, the need for change and correction of lifestyle, long, expensive and complicated treatment that requires considerable effort on the patient and his family, may result decreased quality of life, dissatisfaction, adherence therapy quality of care that ultimately may affect on the prognosis of the disease and in particular atopic dermatitis [3][4].

\section{THE AIM}

The aim of our study was to detect communication between comorbid diseases in children and the level of compliance to therapy.

\section{MATERIALS AND METHODS}

A comparative controlled study in parallel groups, selected by stratified randomization among 67 children with $A D$, in life history or at the time of the study. All patients were informed about all study procedures and gave informed consent to participate in the study.

\section{RESULTS}

Comorbid pathology was observed in 47 children $(70.15 \%$ (95\% CI 0,695-0,707)). The combination of $\mathrm{AD}$ with various allergic diseases observed in 35 children: atopic asthma - in 30 (63.8\% (95\% CI 0,485-0,773)), allergic rhinitis - in $3(4.48 \%$ (95\% CI 0,009-0,125)), allergic conjunctivitis - in $2(2.9 \%$ (95\% CI 0,003-0,103)). Additionally, comorbid conditions were: chronic gastroduodenitis - 40 children $(59.7 \%(95 \% \mathrm{CI}$ $0,470-0,715))$; biliary dyskinesia - 39 children $(62.6 \%(95 \%$ CI 0,500-0,742)); dysmetabolic nephropathy - 20 children (29.8\% (95\% CI 0,192-0,422)); reactive pancreatitis - 30 children (44.7\% (95\% CI 0,326-0,574)); different psychosomatic disorders - 27 children (40.3\% (95\% CI 0,284- 
$0,530)$ ); vegetative-vascular disorders - 18 children $(26.8 \%$ (95\% CI 0,167-0,390)); chronic tonsillitis - 13 children (19.4\% (95\% CI 0,107-0,308)); acute bronchitis - 4 children $(5.97 \%$ (95\% CI 0,016-0,145)); pneumonia - 4 children $(5.97 \%$ (95\% CI 0,016-0,145)); intrauterine infection (TORCH- infection) 28 children $(41.7 \%$ (95\% CI 0,298-0,544)).

It should be noted that the presence of comorbid diseases in children accompanied by more severe AD. Thus in patients with comorbid pathology median SCORAD scale was 19,15 \pm 2,18 , and in children with isolated $\mathrm{AD}-12,60 \pm 1,20$ points.

Mild course of AD noted in 15 children $(75 \%$ (95\% CI 0.509 - 0.913)) without comorbid disease, moderate $\mathrm{AD}$ - in 5 children (25\% (95\% CI 0.087 - 0.491)).

In group with comorbid pathology mild course of $\mathrm{AD}$ was observed in 30 patients $(63.8 \%$ (95\% CI, 0, $482-0.773)$ ), moderate - in 13 children (27.6\% (95\% CI 0.156 - 0.426)), severe - 4 (8.5\% (95\% CI 0.023 - 0, 203)). When comparing statistics $\mathrm{OR}$ severe and moderate flow of $\mathrm{AD}$ in children with comorbid pathology was 1.700 (95\% CI, 0.525-5.500). Inpatient treatment needed 52 patients. The mean duration of hospitalization in children without comorbidities was - 4,20 \pm 1,30 days $(95 \%$ CI 2,58- 5,81), in patients with comorbid disorders average hospital stay was - 6,72 $\pm 2,16$ days ( $95 \%$ CI 6,08 - 7,35). Patients often receive standard therapy, which included a variety of antihistamines, hyposensitization agents, sedatives, enzyme, vitamins, antibacterial, antispasmodic, holeritic preparations, topical therapy of $\mathrm{AD}$, various softening and moisturizing creams, ointments and creams, containing corticosteroids, antibacterial ointment or combined topical steroids. It should be noted that the standard treatment for patients with comorbid pathology was ineffective. The average duration of remission in these patients with $\mathrm{AD}$ to 1 month was pronounced in 7 (14.8\% (95\% CI: 0,062- 0,283)) children; from 1 to 3 months - in 30 (63.8\% (95\% CI, 0, 482 $0.773)$ ), from 4 to 6 months - in $10(21.3 \%$ (95\% CI 0,107 0,356)).

Patients without comorbid disease remission duration of 1 to 3 months was observed in 7 (35\% (95\% CI $0153-0.592))$ cases, 4 to 6 months - 13 (65\% (95\% CI $0407-0.846$ )) cases. When comparing these indicators for groups $\chi 2$ test was 11.897, p <0,01; OR - 6.871 (95\% CI 2.167- 21.794).

Anonymous survey of patients parents was conducted to find out the satisfaction of their assistance and commitment therapy. In the group of patients with comorbid disorders, more than half of mothers $(59.5 \%)$ were not satisfied with the medical care or partially satisfied, compared with patients without concomitant diseases, where the figure was $30 \%$. Thus, the odds ratio was 3.439 (95\% CI 1.122- 10.537) ( Fig. 1), $\mathrm{RR}=1.986$ (95\% CI 1.202 - 3.280) (Fig. 2.).

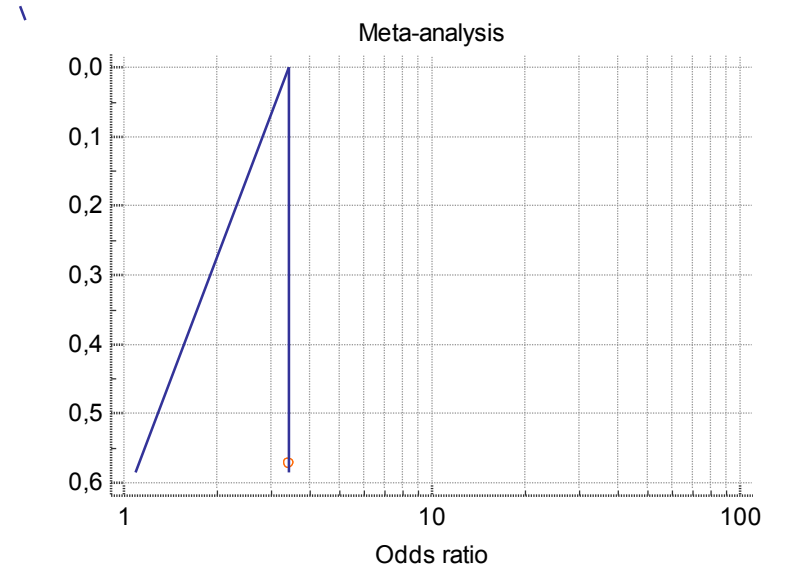

Fig. 1 Results of a meta-analysis of compliance and treatment satisfaction in the comparison groups

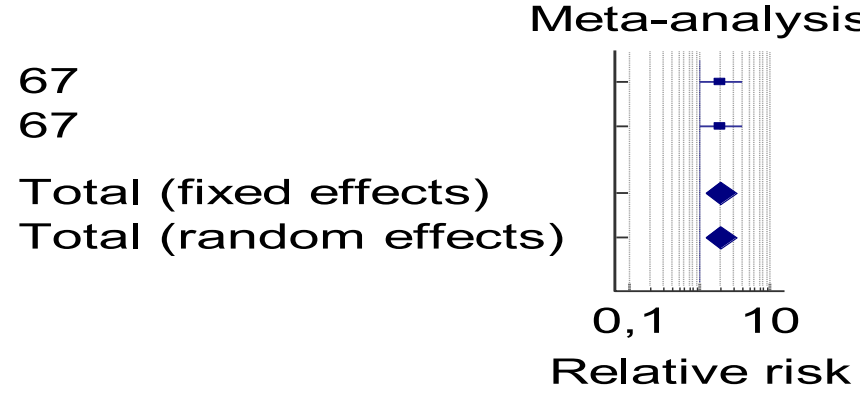

Fig. 2. The result of meta-analysis of compliance in the comparison groups.

Among the reasons for dissatisfaction, respondents pointed to lack of efficacy and duration of treatment, the complexity of the use of drugs, number of drugs and various forms of drugs that had to apply for the relief of the underlying disease, and treatment of comorbidity. 55.7\% of parents said they did not have confidence in the doctor.

\section{CONCLUSIONS}

1. $\mathrm{AD}$ is often accompanied by comorbid diseases in children (70.15\% (95\% CI 0,695-0,707)).

2. Most often $\mathrm{AD}$ accompanies by chronic gastroduodenitis, biliary dyskinesia, atopic asthma, dysmetabolic nephropathy, reactive pancreatitis, different psycho-somatic disorders, vegetative-vascular disorders, chronic tonsillitis, acute bronchitis, pneumonia, intrauterine infection (TORCH infection), allergic rhinitis, allergic conjunctivitis.

3. The presence of comorbid diseases in children accompanied by more severe AD.

4. Compliance was in a group of comorbid disease was significantly lower $(\mathrm{OR}=3.439$ (95\% CI 1.122- 10.537)). Among the reasons for low adherence to treatment can be identified: the duration and lack of effectiveness of the treatment, the complexity of the use of drugs, number of drugs and various forms of drugs that had to apply for the relief of the underlying disease, and treatment of comorbidity. 


\section{REFERENCES}

[1] Spergel JM, Paller AS. Atopic dermatitis and the atopic march. J Allergy Clin Immunol. 2003; 112: S118-S127.

https://doi.org/10.1016/j.jaci.2003.09.033

[2] Burr M.L., Wat D., Evans C. et al. Asthma prevalence in 1973, 1988 and 2003 // Thorax. - 2006. - 61(4). - 296-9.

[3] Ariaev N. L., Shevchenko I. M., Titkova E. V. Atopic dermatitis: a modern view on the problem of medicine of XXI century. Collection of scientific articles with international participation "The survival strategy in terms of bioethics, philosophy and medicine", Chisinau - 2015 Vol. 21 - P.166-169

[4] Stoyeva T. V., Titkova E. V., Reshetilo O. V. Analysis of risk factors for atopic dermatitis in children. 17th International Congress for Medicalis Students and Young Professionals, MEDICALIS 2016, 12-15.05.2016., Cluj-Napoca, Romania 